\title{
Pancreatoduodenectomía de urgencia: Whipple o no Whipple, esa es la cuestión
}

\author{
Emergency pancreatoduodenectomy: Whipple or no Whipple, \\ that is the question
}

Felipe Bernal ${ }^{1}$, Sebastián Sánchez², Mauricio Pedraza², Luis Felipe Cabrera³

${ }^{1}$ Cirugía general, Fundación Salud Bosque, Universidad El Bosque, Bogotá, D.C., Colombia

${ }^{2}$ Interno de Medicina, Universidad El Bosque, Bogotá, D.C., Colombia

${ }^{3}$ Cirugía General, Hospital Santa Clara, E.S.E., Universidad El Bosque, Bogotá, D.C., Colombia

\section{Resumen}

Introducción. La pancreatoduodenectomía, u operación de Whipple, fue descrita por Codivilla en I898 como tratamiento para el cáncer de la cola del páncreas. Con aportes posteriores se ha perfeccionado la técnica, lo cual ha permitido su aplicación en diferentes circunstancias y ha contribuido a la supervivencia de muchos pacientes. Sin embargo, su uso poco frecuente y las dificultades para practicarla, hacen complicado decidir si se utiliza o no la técnica de Whipple.

Caso clínico. Se presenta y analiza el caso de un paciente que ingresó a urgencias por un trauma penetrante de abdomen con una grave lesión pancreatoduodenal; se le practicó de urgencia una operación de Whipple en el servicio de cirugía general de una institución de tercer nivel de Bogotá, y se obtuvieron resultados satisfactorios. Además, se hace una revisión exhaustiva de la literatura científica.

Discusión y conclusiones. La pancreatoduodenectomía de urgencia es una cirugía compleja que supone un reto quirúrgico, en el cual se deben tener en cuenta el estado hemodinámico, el estado ácido-base y la presencia o ausencia de coagulopatía, a la hora de adoptar una conducta definitiva.

Palabras clave: páncreas; duodeno; traumatismos abdominales; heridas penetrantes; pancreaticoduodenectomía.

\begin{abstract}
Introduction: Pancreaticoduodenectomy is a procedure first described by Codivilla in 1898 for the management of pancreatic cancer. With subsequent contributions the technique has been perfected allowing its application in other contexts leading to the survival of many patients. However, the low demand for the procedure and the difficulties in doing so makes the decision of whether to take or not to take the patient to a Whipple surgery a very complex matter.

Case report: We present the case of a patient who arrived at the emergency room with penetrating abdominal trauma with pancreaticoduodenal injury in whom the Whipple procedure was performed urgently by the general surgery service at a third level of care institution in Bogotá, Colombia, with excellent results.
\end{abstract}

Fecha de recibido: 13/07/2017 Fecha de aceptado: 30/08/2017

Correspondencia: Mauricio Pedraza Ciro, MD / Bogotá, D.C., Colombia / Correo electrónico: mpedraza93@gmail.com

Citar como: Bernal F, Sánchez S, Pedraza M, Cabrera LF. Pancreatoduodenectomía de urgencia: Whipple o no Whipple, esa es la cuestión. Rev Colomb Cir. 2018;33:111-8. 
Discussion and conclusions: Emergency pancreatoduodenectomy is a complex procedure that represents a surgical challenge in which the hemodynamic state, the acid base balance and the presence of coagulopathy need to be considered when assuming some definitive conduct.

A comprehensive review of the scientific literature is presented.

Keywords: Pancreas; Duodenum; Abdominal Injuries; Wounds, Penetrating; Pancreaticoduodenectomy.

\section{Introducción}

El trauma pancreatoduodenal de gran complejidad es poco frecuente y, por lo general, requiere de una pancreatoduodenectomía de urgencia, que es un procedimiento poco usual. Esta operación requiere de gran experiencia, conocimiento y habilidad en el manejo de las estructuras del retroperitoneo ${ }^{1,2}$.

La pancreatoduodenectomía está indicada en lesiones pancreáticas, duodenales o mixtas con un grado mayor de III en la escala de la American Association for the Surgery of Trauma (AAST), pues se asocia con una gran mortalidad reportada, de $40 \%$ a $75 \%{ }^{1-5}$. Otras indicaciones para este procedimiento son el sangrado por úlceras, los tumores, las perforaciones y las infecciones.

Por todo lo anterior, en el presente artículo se presenta un caso de pancreatoduodenectomía de urgencia, acompañado de una revisión crítica y exhaustiva de la literatura científica.

\section{Caso clínico}

Se trata de un paciente de sexo masculino de I6 años de edad, sin antecedentes médicos o quirúrgicos de importancia, que fue llevado al servicio de urgencias por un trauma abdominal penetrante por proyectil de arma de fuego, con un trayecto transabdominal y a nivel de los flancos.

$\mathrm{Al}$ momento de la evaluación inicial, se encontraba con alteración del estado de conciencia, pálido, hipoperfundido, con frecuencia cardiaca de 124 latidos por minuto, tensión arterial de $8 \mathrm{I} / 40 \mathrm{~mm} \mathrm{Hg}$ (media=54) y temperatura de $36,4^{\circ} \mathrm{C}$. Se diagnosticó un choque hemorrági- co de grado III y puntaje de 36 en el puntaje de gravedad del trauma ISS (Injury Severity Score).

El paciente fue sometido a una laparotomía exploratoria, en la cual se usó la maniobra de Kocher, y se encontró un trauma pancreatoduodenal de grado V, según AAST, debido a una lesión compleja con pérdida de la unión biliopancreática y más del $75 \%$ de la circunferencia de la segunda porción del duodeno, pérdida del $50 \%$ de la cabeza del páncreas, lesión del confluente esplenoportal, y un trauma hepático de grado II asociado, con hemoperitoneo de I.500 $\mathrm{ml}$ y contaminación de la cavidad peritoneal por contenido intestinal. Con estos hallazgos, se decidió practicar la intervención quirúrgica en dos tiempos.

En el primer tiempo quirúrgico, se llevó a cabo el control de daños con reparo primario de las estructuras vasculares, pancreatoduodenectomía de urgencia y reparo de la primera porción del duodeno, canalización del conducto hepático común y páncreas abandonado, sin ligadura del conducto de Wirsung (figuras I y 2).

Se procedió a la reanimación hipotensiva en la unidad de cuidados intensivos con cristaloides, hemoderivados, ácido tranexámico y retiro progresivo del vasopresor (noradrenalina), y se obtuvo mejoría de los indicadores de perfusión tisular, como el lactato y la saturación venosa de oxígeno.

A las 48 horas, en el segundo tiempo quirúrgico, se reconstruyó el tubo digestivo mediante gastroyeyunostomía sin preservación del píloro, pancreatoyeyunostomía 'telescopada' (sic) y hepaticoyeyunostomía en una sola asa, y se colocó una sonda nasoyeyunal para nutrición entérica (figuras 3 y 4 ). 


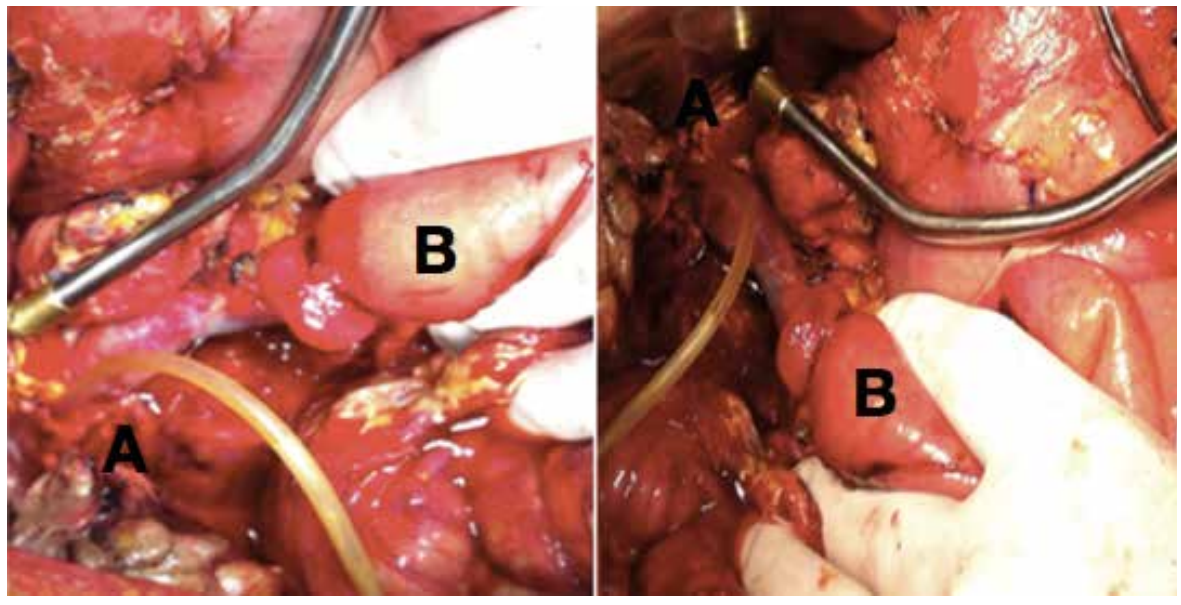

Figura 1. Trauma pancreatoduodenal de grado V, según la AAST; laparotomía abreviada (control de daños) con canalización del conducto hepático común.
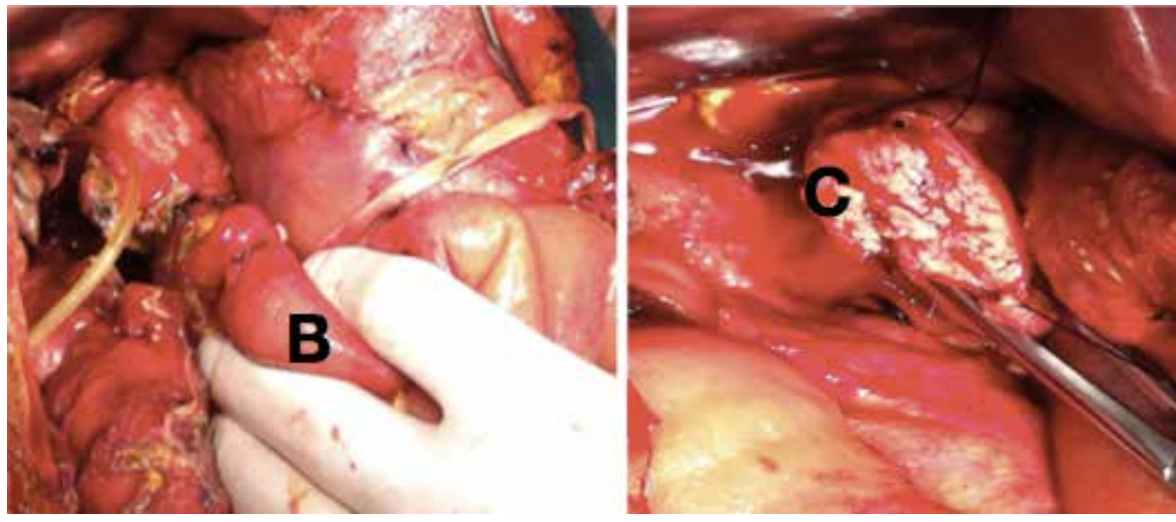

Figura 2. Control de daños: reparo de la primera porción del duodeno y páncreas abandonado sin ligadura del conducto de Wirsung.
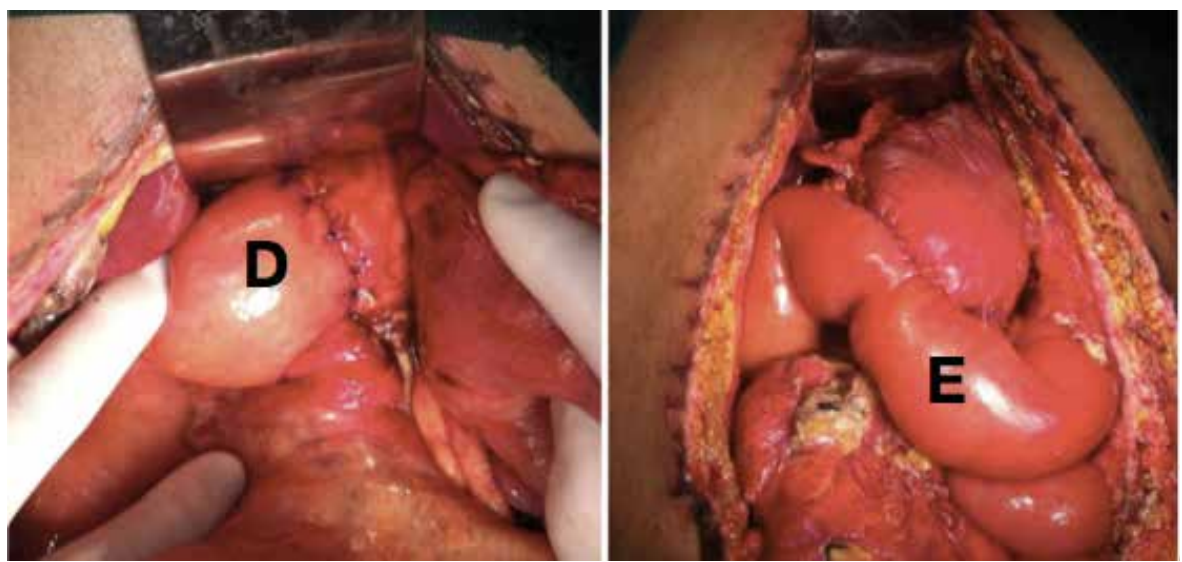

Figura 3. Segunda etapa del control de daños: reconstrucción del tubo digestivo con pancreatoyeyunostomía 'telescopada' (sic) y gastroyeyunostomía sin preservación del píloro 


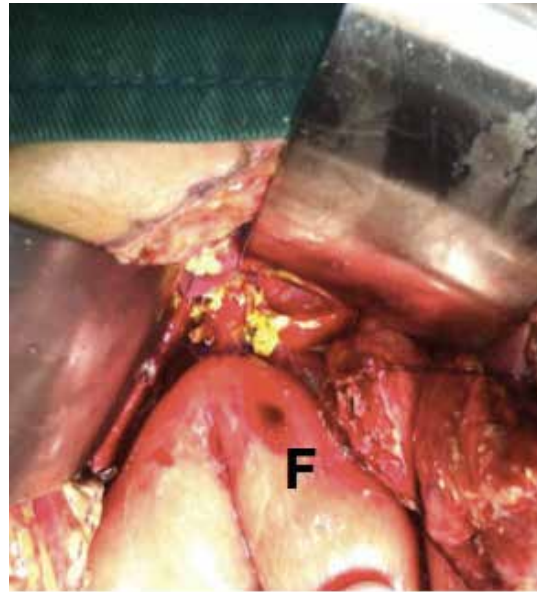

Figura 4. Segunda etapa del control de daños: hepaticoyeyunostomía

Los dos tiempos quirúrgicos sumaron $390 \mathrm{mi}-$ nutos. Presentó un sangrado de $2.000 \mathrm{ml}$, aproximadamente, en la cirugía de control de daños, por lo cual se requirió una transfusión de seis unidades de $200 \mathrm{ml}$ de glóbulos rojos empaquetados. La estancia hospitalaria fue de 20 días. Como única complicación, presentó una fístula pancreática postoperatoria de tipo $\mathrm{A}$, la cual se resolvió con nutrición parenteral total y somatostatina, después de lo cual se dio el alta hospitalaria.

\section{Discusión}

Desde finales del siglo XIX se han practicado pancreatoduodenectomías para el tratamiento de diferentes entidades; sin embargo, hasta el día de hoy, y con los aportes de un gran número de expertos, la técnica continúa siendo un reto para los cirujanos ${ }^{6,7}$.

La primera cirugía de este tipo fue reportada por Codivilla, en I898, en un paciente con un tumor maligno periampular. Se resecaron la cabeza del páncreas, el duodeno, la porción distal del estómago y el colédoco. El resultado final fue la muerte del paciente a los i8 días de la intervención.

Posteriormente, varios cirujanos hicieron esfuerzos importantes al resecar segmentos del páncreas y del duodeno. Sin embargo, no fue sino hasta 1935 cuando Whipple, et al., publicaron una serie de casos de pacientes operados en el Columbia Presbyterian Hospital de Nueva York, a quienes se les practicó resección radical del duodeno y de la cabeza del páncreas como manejo del carcinoma periampular, con resultados favorables. En el mismo año, otros autores acogieron el término de pancreatoduodenectomía de urgencia para el manejo de pacientes con trauma pancreatoduodenal. Hacia 1946, Allen Oldfather Whipple publicó los resultados de su experiencia de Io años, los cuales le llevaron a perfeccionar la técnica que hoy lleva su nombre ${ }^{7-9}$.

La necesidad de una pancreatoduodenectomía de urgencia en trauma depende de los órganos lesionados. El páncreas se ve afectado en menos del $2 \%$ de los traumatismos cerrados de abdomen y, del 20 a $30 \%$, de los penetrantes. La mortalidad de este tipo de lesiones varía entre 9 y $34 \%$, pero aumenta de forma importante cuando el compromiso pancreático está relacionado con lesión de los órganos vecinos ${ }^{8}$. Las estructuras anatómicas más frecuentemente asociadas con la lesión pancreática son: hígado, colon, yeyuno, duodeno e íleon. Sin embargo, lo más común (50\%), es el trauma vascular asociado ${ }^{\text {10. }}$. En los centros de referencia de trauma, se reporta una baja incidencia ( 5 a $9 \%$ ) de lesión pancreatoduodenal, con una alta mortalidad de hasta 38 a $75 \%{ }^{\text {II-13 }}$.

El trauma pancreatoduodenal puede producir diferentes manifestaciones clínicas, que varían desde dolor abdominal hasta peritonitis generalizada, con hemorragia de las vías digestivas altas, vómito, pérdida de la conciencia, inestabilidad hemodinámica, choque y muerte.

A la hora de elaborar la historia clínica, es importante identificar el mecanismo de trauma, ya que puede indicar las posibles estructuras afectadas.

En el caso de un paciente con trauma cerrado de abdomen no valorable y con inestabilidad hemodinámica, debe practicarse una ecografía abdominal enfocada en el trauma (Focused $A b$ dominal Sonography for Trauma, FAST); si los hallazgos son dudosos o hay líquido libre en 
cavidad peritoneal, se debe optar por una laparotomía exploratoria de urgencia y el control de daños.

En un paciente estable, se pueden hacer estudios imagenológicos ${ }^{14}$. La radiografía simple de abdomen puede demostrar escape de aire en el retroperitoneo o en el árbol biliar. La ecografía abdominal es útil para detectar líquido libre en la cavidad peritoneal y confirmar un hemoperitoneo ${ }^{5,14}$. La tomografía computadorizada (TC) debe usarse en pacientes sin inestabilidad hemodinámica y con abdomen no valorable o en aquellos con trauma de alto impacto. No obstante, para que los cambios pancreáticos sean evidentes en la TC, deben haber pasado, por lo menos, I2 horas desde el evento traumático ${ }^{8,14}$. La colangiopancreatografía retrógrada endoscópica permite diagnosticar lesiones del conducto pancreático, cuando se observa fuga del medio de contraste ${ }^{15}$.

En el paciente con trauma penetrante de abdomen, independientemente de su estabilidad hemodinámica, la indicación solía ser una laparotomía exploratoria; así que, en este caso hipotético, los estudios imagenológicos perdían totalmente su validez en la práctica clínica ${ }^{16}$. Sin embargo, la 'evidencia' clínica reciente indica que, en los pacientes con trauma abdominal penetrante, estables y sin signos de irritación peritoneal, es posible practicar una TC abdominal con contraste para evaluar posibles lesiones de los órganos intraabdominales y, de esta forma, evitar una laparotomía de precisión.

Así lo informaron Bennett, et al., en la revista canadiense de cirugía en 20I6, en un estudio prospectivo de casos y controles, con 62 pacientes tratados de forma conservadora y io intervenidos quirúrgicamente. En los casos de trauma abdominal penetrante, observaron que la estabilidad hemodinámica y la ausencia de signos de irritación peritoneal eran predictores fuertes para optar por el manejo médico expectante, con significación estadística $(\mathrm{p}=0,039)$. La estancia hospitalaria disminuyó $\mathrm{I}, 9$ días con respecto a los casos intervenidos quirúrgicamente en la literatura. El marcador de falla de este tratamiento conservador fue la presencia de líquido libre peritoneal demostrado en la TC abdominal con contraste, aunque no se observan lesiones de los órganos intraabdominales ${ }^{17}$.

Las lesiones pancreáticas deben clasificarse de I a V, según la escala de la American Association for the Surgery of Trauma (AAST); las lesiones grado I y II deben tratarse de manera expectante, y las de grado III a V (relevantes para este caso) requieren manejo quirúrgico de urgencia dada su gran mortalidad ${ }^{16,18}$.

Ante lesiones pancreáticas graves, el arsenal terapéutico al alcance del cirujano comprende diferentes procedimientos y técnicas que permiten una aproximación a esta entidad, como la exclusión pilórica, la formación de un divertículo duodenal, procedimiento el cual consiste en canalizar y exteriorizar el drenaje biliar, drenaje del conducto pancreático con tubo en $\mathrm{T}$ y realizar un antrectomía, gastroyeyunostomía, duodenorrafia junto a la canalización del conducto de Wirsung y, finalmente, el procedimiento de Whipple ${ }^{19}$.

El tiempo es crítico en el tratamiento de este tipo de lesiones, ya que la demora de más de 24 horas en la toma de decisiones aumenta la mortalidad en $40 \%$, en comparación con los pacientes tratados en las primeras 24 horas, en quienes se redujo la mortalidad en un II \%, como se ve reflejado en el presente caso ${ }^{4}$.

Como vía de acceso, se sugiere la laparotomía mediana, con exploración meticulosa de toda la cavidad abdominal, incluyendo el retroperitoneo, para intentar dilucidar la extensión y la gravedad de la lesión; además, se debe utilizar la maniobra de Kocher para examinar adecuadamente el duodeno y el confluente biliopancreático.

Debe examinarse el páncreas en toda su extensión, prestando especial atención a hallazgos intraoperatorios como la sección de la cabeza del páncreas, la aparición de líquido pancreático, las perforaciones centrales o las laceraciones graves, con la disrupción masiva de tejido o sin ella, las cuales pueden predecir la presencia de 
una lesión pancreática importante con un alto grado de mortalidad ${ }^{14-16}$.

Se puede descartar una posible lesión del conducto de Wirsung utilizando un disector de Kittner y retractores maleables, evitando producir lesiones iatrogénicas por desvascularización durante la exploración ${ }^{18}$. Además, es esencial revisar todas las porciones del duodeno mediante la maniobra de Cattel-Braasch ${ }^{19}$, generando el mínimo daño colateral al sistema vascular durante la cirugía ${ }^{20}$. Es necesario controlar la hemorragia, la fuga entérica y la pancreática, así como la disrupción biliar, tal como se llevó a cabo en este caso ${ }^{6}$.

Al identificar la ampolla de Vater, se recomienda canalizar con un tubo 5 Fr para practicar una colangiopancreatografía retrógrada $y$, así, descartar fugas y establecer si se requiere una resección pancreática, duodenal o pancreatoduodenal; este procedimiento se puede hacer preservando o resecando el píloro, antes de dar por terminado el primer tiempo quirúrgico.

Es necesario tener en cuenta que la primera cirugía es de control de daños debido a la gravedad del trauma y el mal estado general de este tipo de pacientes. En el segundo tiempo quirúrgico, se lleva a cabo la cirugía definitiva de reconstrucción del tubo digestivo, como fue descrito por Asensio, et al., y se hizo en este paciente ${ }^{1,3,22}$.

Sin embargo, otros factores que se deben tener en cuenta para tomar este tipo de decisiones, son el estado hemodinámico del paciente, el sangrado, el estado ácido-base, la hipotermia y la presencia de coagulopatía. Esto, con el objetivo de establecer si el paciente es o no candidato a una reparación primaria o si requiere un control de daños inicial, como en el presente caso ${ }^{6,13,21-22}$.

Durante el control de daños, se debe detener inicialmente la hemorragia activa y posteriormente la contaminación, con el fin de prevenir la triada de la muerte. Además, en lesiones pancreatoduodenales de alto grado, se debe intentar preservar el parénquima buscando métodos alternativos a la resección, para preservar tanto como sea posible la fisiología del tubo digestivo.
En caso de requerirse la resección, se recomienda una yeyunostomía para nutrición entérica, para proveer el soporte metabólico de forma temprana, lo cual es esencial para la recuperación del paciente. Sin embargo, esta se puede obviar colocando una sonda nasoyeyunal en el segundo tiempo quirúrgico, como se hizo en este caso ${ }^{4,23-25}$.

Las complicaciones más frecuentes de este tipo de intervenciones, son el sangrado masivo con necesidad de transfusión de hemoderivados, las fístulas y los abscesos intraabdominales, las cuales se presentan hasta en $90 \%$ de los pacientes, como se observó en este caso ${ }^{23}$.

No se han reportado estudios controlados aleatorizados en los cuales se compare la cirugía de control de daños con el tratamiento definitivo en una sola intervención quirúrgica ${ }^{19}$. No obstante, la evidencia tomada de series de casos apunta a que, en los pacientes con este tipo de trauma pancreatoduodenal, in extremis, la cirugía en dos tiempos disminuye la mortalidad ${ }^{13,26}$.

Ante la duda de optar por una operación de Whipple convencional o por una pancreatoduodenectomía con preservación del píloro con la técnica de Traverso-Longmire, se debe tener en cuenta el grado de compromiso traumático de los tejidos para establecer si se puede preservar el píloro, ya que, según la literatura científica, los tiempos quirúrgicos, el sangrado, la necesidad de transfusión y la mortalidad, no presentan diferencias estadísticas significativas entre ambos abordajes.

Sin embargo, existe una diferencia trascendental en el desarrollo de retardo en el vaciamiento gástrico, el cual es más frecuente en la pancreatoduodenectomía con preservación del píloro, lo cual repercute en un mayor tiempo de estancia hospitalaria por la tardanza en la tolerancia a la vía oral, aunque a largo plazo se conserva mejor la fisiología gastrointestinal con la técnica de Traverso-Longmire ${ }^{21,27}$. Teniendo en cuenta lo anterior, en el presente caso se practicó una pancreatoduodenectomía convencional sin preservación de píloro. 
Aunque el tiempo promedio de hospitalización reportado es de 24 días, en este paciente fue de 20 días, lo cual se debió posiblemente a la oportuna cirugía temprana de control de daños y a una adecuada reanimación hipotensiva en la unidad de cuidados intensivos ${ }^{16,18,27}$.

\section{Conclusiones}

Las lesiones traumáticas pancreatoduodenales complejas producen una gran mortalidad. $\mathrm{Su}$ escasa incidencia hace difícil la decisión de optar por una intervención quirúrgica definitiva o por el manejo no operatorio; además, se traduce en pocos cirujanos expertos, lo cual se aúna a los grandes desafíos técnicos propios del procedimiento de Whipple.

Aunque la 'evidencia' no es definitiva, según la experiencia de nuestra institución, y los estudios observacionales y reportes de casos, este tipo de trauma amerita una cirugía de control de daños con posterior reanimación hipotensiva en la unidad de cuidados intensivos, antes de decidirse por un tratamiento quirúrgico definitivo ${ }^{28,29}$.

\section{Referencias}

I. Asensio JA, Petrone P, Roldán G, Pak-art R, Salim A. Pancreatic and duodenal injuries. Complex and lethal. Scand J Surg. 2002;9I:8I-6.

2. Vane D, Kiankhooy A, Sartorelli K, Vane J. Initial resection of potentially viable tissue is not optimal treatment for grades II-IV pancreatic injuries. World J Surg. 2009;33:22I-7.

3. Asensio JA, Demetriades D, Berne TV, Berne JD, Falabella A, Gómez H, et al. A unified approach to the surgical exposure of pancreatic and duodenal injuries. Am J Surg. 1997;174:54-60.

4. Leitmeyer K, Adlhoch C, Sharma A. Management of pancreaticoduodenal injuries. Epidemiology. 20I6;27:743-5I.

5. Blocksom JM, Tyburski JG, Sohn RL, Williams M, Harvey E, Steffes CP, Carlin AM.et al. Prognostic determinants in duodenal injuries. Am Surg. 2004;70:248-55.

6. Mansour MA, Moore JB, Moore EE, Moore FA. Conservative management of combined pancreatoduodenal injuries. Am J Surg. 1989;I58:53I-5.

7. Are C, Dhir M, Ravipati L. History of pancreaticoduodenectomy: Early misconceptions, initial milestones and the pioneers. HPB (Oxford). 20II;13:377-84.
8. Debi U, Kaur R, Prasad KK, Sinha SK, Sinha A, Singh K. Pancreatic trauma: A concise review. World J Gastroenterol. 2013;19:9003.

9. Schnelldorfer T. Alessandro Codivilla and the first pancreatoduodenectomy. Archives of Surgery. 2009;I44:II79.

Io. Potoka DA, Gaines BA, Leppäniemi A, Peitzman AB. Management of blunt pancreatic trauma: What's new? Eur J Trauma Emerg Surg. 2015;41:239.

II. Oreskovich MR, Carrico CJ. Pancreatoduodenectomy for trauma: a viable option? Am J Surg. 1984;147:618-23.

12. Chrysos E, Athanasakis E, Xynos E. Pancreatic trauma in the adult: Current knowledge in diagnosis and management. Pancreatology. 2002;2:365-78.

I3. Krige JE, Nicol AJ, Navsaria PH. Emergency pancreatoduodenectomy for complex injuries of the pancreas and duodenum. HPB (Oxford). 20I4;16:IO43-9.

I4. Asensio JA, Petrone P, Roldán G, Kuncir E, Demetriades D. Pancreaticoduodenectomy: A rare procedure for the management of complex pancreaticoduodenal injuries. J Am College Surg. 2003;197:937-42.

I5. Lissidini G, Prete FP, Piccinni G, Gurrado A, Giungato $\mathrm{S}$, Prete $\mathrm{F}$, et al. Emergency pancreaticoduodenectomy: When is it needed? A dual non-trauma centre experience and literature review. Int J Surg. 20I5;2I(Suppl.I):S83-8.

I6. Tianjin C, Hong G, Zhangying W, Dean M, Jianping G, Can G. The diagnosis and treatment of pancreas injury: A report of 26 cases. J Traumatic Stress Disor Treat. 2016;5. doi:I0.4172/2324-8947.IO00I53

I7. Bennett S, Amath A, Knight H, Lampron J. Conservative versus operative management in stable patients with penetrating abdominal trauma: The experience of a Canadian level I trauma centre. Can J Surg. 20I6;59:3I7-2I.

I8. Berne CJ, Donovan AJ, Hagen WE. Combined duodenal pancreatic trauma the role of end-to-side gastrojejunostomy. Arch Surg. 1968;96:712-22.

I9. Cirocchi R, Montedori A, Farinella E, Bonacini I, Tagliabue L, Abraha I. Damage control surgery for abdominal trauma. Cochrane Database Syst Rev. 2013;(3):CDoo7438. doi: IO.IOO2/I465I858. CDoo7438.pub3

20. Pereira J, Aveiro D, Constantino J, Oliveira A, Pinheiro LF. Trauma pancreatoduodenectomy: How and why? Case Rep Int. 20I5;4:57-6I

2I. Hüttner FJ, Fitzmaurice C, Schwarzer G, Seiler CM, Antes G, Büchler MW, et al. Pylorus-preserving pancreatico-duodenectomy (pp Whipple) versus pancreaticoduodenectomy (classic Whipple) for surgical treatment of periampullary and pancreatic carcinoma. Cochrane Database Syst Rev. 2016(2):CDoo6053.

22. Asensio JA, Demetriades D, Hanpeter DE, Gambaro E, Chahwan S. Management of pancreatic injuries. Curr Probl Surg. 1999;36:325. 
23. Birkmeyer JD, Stukel TA, Siewers AE, Goodney PP, Wennberg DE, Lucas FL. Surgeon volume and operative mortality in the United States. N Engl J Med. 2003;349:2II7.

24. Rotondo MF, Schwab CW, McGonigal MD, Phillips GR 3rd, Fruchterman TM, Kauder DR, et al. "DAMAGE control": an approach for improved survival in exsanguinating penetrating abdominal injury . J Trauma. 1993;35:375-83.

25. Koniaris LG. Role of pancreatectomy after severe pancreaticoduodenal trauma. J Am Coll Surg. 2004;198:677-8.

26. van der Wilden GM, Yeh D, Hwabejire JO, Klein EN, Fagenholz PJ, King DR, et al. Trauma Whipple:
Do or don't after severe pancreaticoduodenal injuries? An analysis of the National Trauma Data Bank (NTDB). World J Surg. 20I4;38:335-340.

27. Asensio A, Petrone P, Kimbrell B, Kuncir E. Trauma duodenal. Técnica y manejo. Rev Colomb Cir. 2006;2I:4-I4.

28. Gulla A, Tan WP, Pucci MJ, Dambrauskas Z, Rosato EL, Kaulback KR, et al. Emergent pancreaticoduodenectomy: A dual institution experience and review of the literature. J Surg Research. 20I4;I86:I-6.

29. Petrone P, Moral S, González M, Ceballos J, Marini CP. Pancreatic trauma: Management and literature review. Cir Esp. 20I7;95:I23-30. 